\title{
Cytotoxic Lymphocytes Target HIV-1 Gag Through Granzyme M-Mediated Cleavage
}

\author{
Elisa Saccon ${ }^{1}$, Flora Mikaeloff ${ }^{1}$, Pol Figueras Ivern ${ }^{2}$, Ákos Végvári ${ }^{3}$, \\ Anders Sönnerborg ${ }^{1,2,4}$, Ujjwal Neogi ${ }^{1,4}$ and Robert van Domselaar ${ }^{2 *}$ \\ 1 Division of Clinical Microbiology, ANA Futura Laboratory, Department of Laboratory Medicine, Karolinska Institutet, \\ Stockholm, Sweden, ${ }^{2}$ Division of Infectious Diseases, ANA Futura Laboratory, Department of Medicine Huddinge, Karolinska \\ Institutet, Stockholm, Sweden, ${ }^{3}$ Division of Chemistry I, Department of Medical Biochemistry and Biophysics, Karolinska \\ Institutet, Stockholm, Sweden, ${ }^{4}$ Molecular Microbiology and Immunology, University of Missouri, Columbia, MO, United States
}

\section{OPEN ACCESS}

Edited by: Julia G. Prado, IrsiCaixa, Spain

Reviewed by:

Julian Pardo,

Fundacion Agencia Aragonesa para la Investigacion y el Desarrollo, Spain Samuel Ken-En Gan, Experimental Drug Development Centre (EDDC), Singapore Yasien Sayed, University of the Witwatersrand, South Africa

*Correspondence:

Robert van Domselaar robert.van.domselaar.1@ki.se

Specialty section: This article was submitted to Viral Immunology, a section of the journal

Frontiers in Immunology

Received: 18 February 2021 Accepted: 31 March 2021 Published: 19 April 2021

Citation:

Saccon E, Mikaeloff F,

Figueras Ivern P, Végvári Á,

Sönnerborg A, Neogi $U$ and van Domselaar R (2021)

Cytotoxic Lymphocytes Target HIV-1 Gag Through Granzyme M-Mediated Cleavage.

Front. Immunol. 12:669347. doi: 10.3389/fimmu.2021.669347
Untreated HIV-1 infection leads to a slow decrease in CD4 ${ }^{+} \mathrm{T}$ cell lymphocytes over time resulting in increased susceptibility to opportunistic infections (acquired immunodeficiency syndrome, AIDS) and ultimately death of the infected individual. Initially, the host's immune response controls the infection, but cannot eliminate the HIV-1 from the host. Cytotoxic lymphocytes are the key effector cells in this response and can mediate crucial antiviral responses through the release of a set of proteases called granzymes towards HIV-1infected cells. However, little is known about the immunological molecular mechanisms by which granzymes could control HIV-1. Since we noted that HIV-1 subtype C (HIV-1C) Gag with the tetrapeptide insertion PYKE contains a putative granzyme M (GrM) cleavage site (KEPL) that overlaps with the PYKE insertion, we analyzed the proteolytic activity of GrM towards Gag. Immunoblot analysis showed that GrM could cleave Gag proteins from HIV$1 \mathrm{~B}$ and variants from HIV-1C of which the Gag-PYKE variant was cleaved with extremely high efficiency. The main cleavage site was directly after the insertion after leucine residue 483. GrM-mediated cleavage of Gag was also observed in co-cultures using cytotoxic lymphocytes as effector cells and this cleavage could be inhibited by a GrM inhibitor peptide. Altogether, our data indicate towards a noncytotoxic immunological mechanism by which GrM-positive cytotoxic lymphocytes target the HIV-1 Gag protein within infected cells to potentially control HIV-1 infection. This mechanism could be exploited in new therapeutic strategies to treat HIV-1-infected patients to improve immunological control of the infection.

Keywords: cytotoxic lymphocyte, granzyme M, Gag (HIV-1), HIV-1, immune control

\section{INTRODUCTION}

A key component of both the innate and adaptive immune response against viruses is represented by cell-mediated cytotoxicity, mediated by cytotoxic lymphocytes, through the release of granules containing perforin and granzymes. Granzymes are a family of serine proteases that can be expressed by cytotoxic lymphocytes, such as natural killer (NK) cells and $\mathrm{CD}^{+} \mathrm{T}$ cells. During the 
early phases of infection, granzymes enter the virus-infected target cells, facilitated by pore-forming protein perforin, and mediate antiviral effects through cleavage of host and/or viral proteins. In humans, five granzymes are encoded, namely GrA, $\mathrm{GrB}, \mathrm{GrH}, \mathrm{GrK}$, and GrM (1-4). These five granzymes differ in their antiviral functions through their difference in substrate specificity (5). Specifically, GrM cleaves after a leucine or methionine and its four amino acid consensus substrate motif is $\mathrm{KEPL}(6,7)$. GrM can mediate antiviral effects through induction of apoptosis or by inhibiting viral replication independent of cell death $(4,8,9)$. Noncytotoxic functions of granzymes have been shown to be of importance in controlling chronic and latent infections, such as herpes simplex virus type I and cytomegalovirus infections (8-10). On the other hand, little is known about the role of granzymes in HIV-1 infection. A recent study showed that GrM protein expression in HIV-1 specific $\mathrm{CD}^{+} \mathrm{T}$ cells from HIV-1 infected individuals was the highest compared to expression of the other granzymes (11). Furthermore, in the study by Clayton et al., killing of HIV-1infected $\mathrm{CD}^{+} \mathrm{T}$ lymphocytes by HIV-1-specific $\mathrm{CD}^{+} \mathrm{T}$ lymphocytes was not mediated through granzyme $\mathrm{B}$, because killing still occurred in the presence of a synthetic GrB inhibitor, and the target cells expressed the GrB-inhibitor protein SerpinB9. Which other granzymes could be responsible for the killing of the HIV-1-infected $\mathrm{CD} 4^{+} \mathrm{T}$ cells and whether these granzymes employ noncytotoxic antiviral functions remain unclear. This granzyme-mediated antiviral mechanism could be exploited or boosted in HIV-1-infected individuals to provide a better immunological control of their infection.

Among all HIV-1 subtypes, HIV-1 subtype B (HIV-1B) is the most prevalent in most high-income countries. However, subtype C (HIV-1C) causes over $50 \%$ of all HIV-1 infections worldwide and has become increasingly prevalent in Europe (12, 13). Previously, a tetrapeptide insertion PYKE within a relatively conserved region of the viral Gag protein $\left(\mathrm{Gag}_{\text {PYKEi }}\right.$ ) was identified in a subset of HIV-1C-infected individuals (14). The HIV-1 Gag protein mediates the assembly, budding, and maturation of new virions (15). The PYKE motif is important for the interaction of the viral Gag to host cell protein ALIX that facilitates viral budding. Furthermore, insertion of this motif correlated with enhanced viral fitness $(16,17)$. Interestingly, HIV-1C Gag with the PYKE insertion $\left(\mathrm{Gag}_{\mathrm{PYKEi}}\right)$ contains the GrM consensus substrate motif KEPL overlapping the PYKE motif, whereas HIV-1B and HIV-1C without the tetrapeptide insertion only contain the proline and leucine residues at this site

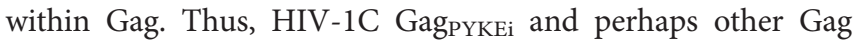
variants are putative GrM substrates, and cleavage by GrM could then potentially interfere with budding and infectiousness of new HIV-1 virions.

In this study, we further investigated the role of GrM in HIV1 infection. First, we evaluated the proteolytic potential of GrM to target the Gag protein from HIV-1 subtypes B (HIV-1B) and $\mathrm{C}$ (HIV-1C), including HIV-1C with or without the tetrapeptide insertion. Then, we assessed whether GrM can target the Gag protein in an in vitro cell model. Finally, we examined the gene and protein expression of GrM within peripheral blood mononuclear cells (PBMCs) of a cohort of HIV-1 infected individuals. Specifically, we compared the proteotranscriptomic profiles of Elite controllers (ECs) to viral progressors (VPs) and uninfected individuals (HC), to assess whether differences in GrM levels could be an underlying immunological mechanism by which ECs can control their HIV-1 infection in the absence of antiretroviral therapy.

\section{MATERIAL AND METHODS}

\section{Cell Culture}

Cells were cultured in $5 \% \mathrm{CO}_{2}$ at $37^{\circ} \mathrm{C}$. HEK293FT cells were maintained in Dulbecco's modified Eagle medium (DMEM, Gibco/ThermoFisher Scientific, USA) supplemented with $10 \%$ fetal bovine serum (FBS, Sigma, USA), $2 \mathrm{mM}$ L-glutamine (Sigma, USA), $0.1 \mathrm{mM}$ MEM Non-Essential Amino Acids (Gibco/ThermoFisher Scientific, USA), and 20 units/mL penicillin combined with $20 \mu \mathrm{g} / \mathrm{mL}$ streptomycin (Sigma, USA). HeLa cells (\#ATCC CCL-2) were maintained in DMEM supplemented with $10 \%$ FBS and 20 units $/ \mathrm{mL}$ penicillin combined with $20 \mu \mathrm{g} / \mathrm{mL}$ streptomycin. KHYG-1 cells (\#ACC 725, DSMZ, Germany) were maintained in Roswell Park Memorial Institute 1640 (RPMI, Sigma, USA) medium supplemented with 10\% FBS, 25 mM HEPES, 20 units $/ \mathrm{mL}$ penicillin and $20 \mu \mathrm{g} / \mathrm{mL}$ streptomycin, and 100 units $/ \mathrm{mL}$ of recombinant human interleukin-2 (IL-2, PeptroTech, Sweden).

\section{Plasmids}

pCR3.1/HIV1B-Gag-mCherry (HIV-1B Gag) was a kind gift from Dr. Paul Bieniasz (The Rockefeller University, USA). pCR3.1/ HIV1C-Gag-mCherry variants with or without PYKE or PYQE tetrapeptide insertions were described previously (16). The

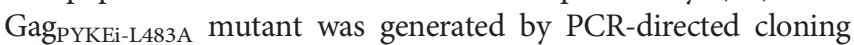
using pCR3.1/HIV-GagPYKEi-mCherry as template. All other Gag $_{\text {PYKEi }}$ mutants were generated by PCR-directed cloning using the L483A mutant as template. PCR was performed using Phusion DNA polymerase (NEB) and corresponding primers

TABLE 1 | Primers used for mutagenesis of Gag.

\begin{tabular}{lll}
\hline Gag mutation & \multicolumn{1}{c}{ Forward primer $^{\mathbf{a}}$} & \multicolumn{1}{c}{ Reverse primer } \\
\hline L101A & 5'-GCC GAC AAA ATT GAG GAA GAG CAG AAT GAG-3' & 5'-AGC CTC CT TGT GTC TाT GAC CTC TAT C-3' \\
L145A & 5'-GCG ACT CCA AGG ACT CTG AAT GCC-3' & 5'-TGG CTG ATG GAC CAT CTG CCC-3' \\
L483A & 5'-GCG ACG AGC CTC AAA AGC TTG TTC-3' & 5'-AGG CTC CT ATA GGG ACC CTG G-3' \\
L495A & 5'-GCG TCC CAG GGC GGC C-3' & 5'-TGG ATC GCT TCC GAA CAA GCT TाT G-3'
\end{tabular}

${ }^{a}$ The bold underlined nucleotides represent the changed nucleotides required to mutate a leucine into an alanine within the plasmid template. 
(Table 1). Linear amplicons were circularized using T4 polynucleotide kinase and T4 ligase. DNA sequences from all constructs were verified by Sanger sequencing.

\section{Antibodies and Reagents}

Rabbit monoclonal antibody against GFP (EPR14104, ab183734), rabbit polyclonal antibody against mCherry (ab167453), and rabbit polyclonal to HIV-1 gag (p55 + p24 + p17, ab63917) were purchased from Abcam (UK). Horse-radish peroxidaseconjugated goat anti-rabbit was obtained from Agilent Dako (USA). NuPAGE ${ }^{\mathrm{TM}} 4-12 \%$ Bis-Tris Protein Gels and iBlot ${ }^{\mathrm{TM}}$ Transfer Stacks were purchased from ThermoFisher Scientific (USA). Immunoblotted proteins were detected using the Enhanced Chemiluminescence detection system (Amersham, UK) and ChemiDoc XRS+ (Bio-Rad, USA). Pan-caspase inhibitor zVAD-FMK was purchased from Enzo Life Sciences (USA) and GrM inhibitor AcKVPL-CMK from Peptanova (Germany).

\section{GrM Cleavage Assay}

Purified recombinant catalytically active human GrM and the catalytically inactive GrM variant (GrM-SA) were a kind gift from Dr. N. Bovenschen (University Medical Center Utrecht, The Netherlands). HEK293FT cells $\left(4 \times 10^{6}\right)$ were seeded into a $10 \mathrm{~cm}$ cell culture dish one day prior to transfection. Then, cells were transfected with one of the Gag-mCherry variants $(8 \mu \mathrm{g})$ using FuGene HD according to the manufacturer's instructions (Promega, USA) in a 3:1 ratio with DNA. One day post-transfection, cell-free protein lysates were generated by washing cells three times in PBS (Karolinska Universitetssjukhuset Huddinge, Sweden) and subsequent lysis in PBS by three cycles of freeze-thawing using liquid nitrogen. Samples were centrifuged at $18,000 \mathrm{x} g$ for $10 \mathrm{~min}$ at $4^{\circ} \mathrm{C}$, supernatant was collected, aliquoted and stored at $-20^{\circ} \mathrm{C}$. Protein concentration was determined by DC protein assay (BioRad, USA). Lysates $(10 \mu \mathrm{g})$ were incubated with indicated concentrations of either GrM or GrM-SA supplemented with a Tris-buffer (50 mM Tris- $\mathrm{HCl} \mathrm{pH} 7.4$ and $150 \mathrm{mM} \mathrm{NaCl}$ ) to a total volume of $12 \mu \mathrm{L}$ for 4 hours (or otherwise indicated) at $37^{\circ} \mathrm{C}$. Then, $4 \mathrm{x}$ Laemmli buffer was added, samples were incubated at $95^{\circ} \mathrm{C}$ for $10 \mathrm{~min}$ and subjected to immunoblotting. ImageLab software (Bio-Rad, USA) was used to quantify protein band intensities and GraphPad Prism was used to plot values in a graph.

\section{KHYG-1 and IL-2-Activated PBMC Killer Cell Assay}

PBMCs were isolated from buffy coats of anonymous donors using Ficoll density centrifugation, aliquoted and stored in liquid nitrogen. A frozen aliquot of PBMCs was thawed and incubated for 4 days in RPMI 1640 medium (Gibco, USA) supplemented with $5 \%$ human $\mathrm{AB}$ serum (Sigma, USA) and 1000 units/mL of recombinant human IL-2. HeLa cells $\left(2 \times 10^{6}\right)$ were collected and transfected with pCR3.1/HIV-GagPYKEi-mCherry $(7.5 \mu \mathrm{g})$ and pEGFP-N1 $(2.5 \mu \mathrm{g})$ and directly seeded into a 96-well plate (20,000 cells per well). When the pan-caspase inhibitor zVADFMK was used, seeded cells were incubated with $50 \mu \mathrm{M}$ of zVAD-FMK or only DMSO (0.5\%). One day post-transfection, transfected HeLa cells were challenged with either KHYG-1 cells or IL-2-activated PBMCs in indicated effector:target (E:T) ratios for in $5 \% \mathrm{CO}_{2}$ at $37^{\circ} \mathrm{C}$. When GrM inhibitor AcKVPL-CMK was used, KHYG-1 cells were pre-treated with $100 \mu \mathrm{M}$ of AcKVPLCMK for 1 hour, before the co-culture with target cells was started. After indicated times, cells were washed twice with PBS and directly lysed in $2 \mathrm{x}$ Laemmli buffer. Samples were incubated at $95^{\circ} \mathrm{C}$ for $10 \mathrm{~min}$. and subjected to immunoblotting. ImageLab software was used to quantify protein band intensities and GraphPad Prism was used to plot values in a graph.

\section{Gene Expression Analysis on Patient Samples}

Whole blood was obtained from HIV-1-negative donors (HC, $\mathrm{n}=16$ ), untreated HIV-1-infected individuals without viremia (Elite Controllers, EC, $\mathrm{n}=19$ ), and treatment naïve HIV-1-infected patients with viremia (Viremic Progressors, VP, $n=17$ ) as described before (18). The criteria to be classified as EC are that the patient has been diagnosed as HIV-1 seropositive for over a year with $\geq 3$ consecutive viral loads of $<75$ viral RNA copies $/ \mathrm{mL}$ (with all previous viral loads below 1000 copies/mL) or has been HIV-1 seropositive for over 10 years with a minimum of two viral loads (and in total $90 \%$ of all viral loads) of 400 copies $/ \mathrm{mL}$. In case of the ECs, the viral load was below the detection limit $(<20$ copies $/ \mathrm{mL})$ at time of sampling. PBMCs were isolated from whole blood through Ficoll density gradient centrifugation (Ficoll-Plaque PLUS, GE Healthcare, USA), aliquoted and stored in liquid nitrogen. RNA sequencing on extracted RNA from PBMCs was performed as described previously (18). Then, data was extracted using Ensembl transcript IDs (Table 2) and normalized transcript level values for each patient within each patient group were plotted in graphs.

\section{Protein Expression Analysis on Patient Samples Using LC-MS/MS}

Proteomic analysis was performed on extracted proteins from PBMCs of nine male individuals from each group. Cell pellets were suspended in $40 \mu \mathrm{L}$ of $0.1 \%$ ProteaseMAX ${ }^{\mathrm{TM}}$ (Promega,

TABLE 2 | Transcript and protein IDs used for extracting data regarding transcript and protein levels for all human granzymes and perforin.

\begin{tabular}{|c|c|c|c|c|c|}
\hline Protein name & Gene name & Transcript ID & bp & UniProt & aa \\
\hline Granzyme A & GZMA & ENST00000274306.7 (GZMA-201) & 896 & P12544 & 262 \\
\hline Granzyme B & GZMB & ENST00000216341.9 (GZMB-201) & 891 & P10144 & 247 \\
\hline Granzyme H & GZMH & ENST00000216338.9 (GZMH-201) & 936 & P20718 & 246 \\
\hline Granzyme K & GZMK & ENST00000231009.2 (GZMK-201) & 1509 & P49863 & 264 \\
\hline Granzyme M & GZMM & ENST00000264553.6 (GZMM-201) & 924 & P51124 & 257 \\
\hline Perforin & PRF1 & ENST00000373209.2 (PRF1-201) & 2492 & P14222 & 555 \\
\hline
\end{tabular}

bp, base pairs; aa, amino acids. 
USA) and $4 \mathrm{M}$ urea in $50 \mathrm{mM}$ ammonium bicarbonate and $10 \%$ acetonitrile (ACN). First, the samples were sonicated with pulse $2 / 2$ at a $20 \%$ amplitude for 1 min using Vibra-Cell ${ }^{\mathrm{TM}}$ probe (Sonics \& Materials, Inc., Newtown, CT). This was followed with sonication in a water bath for $5 \mathrm{~min}$. Then, lysates were vortexed and centrifuged for $5 \mathrm{~min}$ at 13,000 rpm after which the supernatant was collected. The supernatants yielded 3-95 $\mu \mathrm{g}$ proteins.

Ten $\mu$ g of each sample (except for the samples we designated as $\mathrm{EC} 1(7 \mu \mathrm{g}), \mathrm{HC} 13(3 \mu \mathrm{g}), \mathrm{HC} 17(3 \mu \mathrm{g}), \mathrm{VP} 3(8 \mu \mathrm{g})$ and VP10 $(3 \mu \mathrm{g})$ ) were subjected to proteolytic digestion following reduction in $6 \mathrm{mM}$ dithiothreitol at $37^{\circ} \mathrm{C}$ for $60 \mathrm{~min}$ and alkylation in $22 \mathrm{mM}$ iodoacetamide for $30 \mathrm{~min}$ at room temperature in the dark. Trypsin was added as enzyme to a protein ratio of $1: 50$ and digestion was carried out at $37^{\circ} \mathrm{C}$ overnight. Tryptic peptides were cleaned on Thermo Scientific $^{\mathrm{TM}}$ HyperSep $^{\mathrm{TM}}$ C18 Filter Plate, bed volume $40 \mu \mathrm{L}$ (ThermoFisher Scientific, USA) and dried in a centrifugal concentrator (Genevac ${ }^{\mathrm{TM}}$ miVac, Fisher Scientific, USA).

Thermo Scientific ${ }^{\text {TM }}$ TMT10plex ${ }^{\text {TM }}$ isobaric label reagents (ThermoFisher Scientific, USA) in $100 \mu \mathrm{g}$ aliquots were dissolved in $30 \mu \mathrm{L}$ dry $\mathrm{ACN}$, scrambled and mixed with the digested samples solubilized in $70 \mu \mathrm{L}$ triethylammonium bicarbonate, followed by incubation at $22^{\circ} \mathrm{C}$ for $2 \mathrm{~h}$ at 550 $\mathrm{rpm}$. The reaction was quenched with $12 \mu \mathrm{L}$ of $5 \%$ hydroxylamine at $22^{\circ} \mathrm{C}$ for $15 \mathrm{~min}$ at $550 \mathrm{rpm}$. The labeled samples were pooled and dried in a centrifugal concentrator.

The TMT-labeled tryptic peptides were dissolved in $20 \mu \mathrm{L}$ of $2 \%$ ACN/0.1\% formic acid. Reversed-phase liquid chromatography was performed on a Thermo Scientific ${ }^{\mathrm{TM}}$ EASY-nLC 1000 system (ThermoFisher Scientific, USA) on-line coupled to a Q Exactive ${ }^{\mathrm{TM}}$ Plus Hybrid Quadrupole-Orbitrap ${ }^{\mathrm{TM}}$ mass spectrometer (ThermoFisher Scientific, Germany). Samples were injected onto a $50 \mathrm{~cm}$ long C18 Thermo Scientific ${ }^{\mathrm{TM}}$ EASY-Spray ${ }^{\mathrm{TM}}$ column (ThermoFisher Scientific, USA) and separated with the following gradient: $4-26 \% \mathrm{ACN}$ in $180 \mathrm{~min}, 26-95 \% \mathrm{ACN}$ in $5 \mathrm{~min}$, and $95 \%$ $\mathrm{ACN}$ for $8 \mathrm{~min}$ at $300 \mathrm{~nL} / \mathrm{min}$ flow rate. Mass spectrometric data acquisition was comprised of one survey mass spectrum in $\mathrm{m} / z 350$ to 1600 range, acquired with 140,000 (at $\mathrm{m} / z$ 200) resolution, followed by higher energy collision dissociation (HCD) fragmentations of the 16 most intense precursor ions with $2+$ and $3+$ charge state, using $60 \mathrm{~s}$ dynamic exclusion. The tandem mass spectra were acquired with 70,000 resolution, targeting $2 \times 10^{5}$ ions, using $\mathrm{m} / z 2.0$ isolation width and $33 \%$ normalized collision energy.

The raw data files were directly loaded in Thermo Scientific ${ }^{\text {TM }}$ Proteome Discoverer ${ }^{\mathrm{TM}}$ v2.2 (ThermoFisher Scientific, USA) and searched against human SwissProt protein databases $(21,008$ entries) using the Mascot Server 2.5.1 search engine (Matrix Science Ltd., UK). Search parameters were chosen as follows: up to two missed cleavage sites for trypsin, mass tolerance of precursor and $\mathrm{HCD}$ fragment ions at $10 \mathrm{ppm}$ and $0.05 \mathrm{Da}$, respectively. Dynamic modifications of oxidation on methionine, deamidation of asparagine and glutamine and acetylation of $\mathrm{N}$-termini were set. For quantification, both unique and razor peptides were requested. Protein raw data abundance was first filtered with an in-house script and quantile normalized with NormalizerDE v1.4.0 (19).
Histogram was used to assess that the distribution follows a normal law. The batch effect of multiple TMT experiments was removed using the ComBat function of the sva R package v3.34.0 (20). Differential expression analysis was performed with $\mathrm{R}$ package limma v3.42.2 to determine protein abundances (21). BenjaminiHochberg (BH) adjustment and 0.05 FDR cut-off was applied. Finally, data were extracted using the Uniprot IDs (Table 2) and normalized protein abundances for each patient within each patient group were plotted in graphs.

\section{RESULTS}

\section{GrM Targets Various HIV-1 Gag Variants}

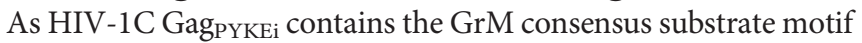

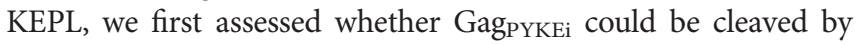
GrM. Since the predicted cleavage site is within the P6 late domain of Gag, we used an HIV-1C Gag PYKEi $_{\text {cDNA construct }}$ with mCherry fused to its C-terminus. We prepared cell-free protein lysates with overexpressed mCherry-tagged Gag ${ }_{\mathrm{PYKEi}}$, incubated them with GrM and assessed GrM-mediated Gag $_{\text {PYKEi }}$ cleavage by immunoblotting using antibodies against either mCherry or Gag. Whereas the catalytically inactive GrM

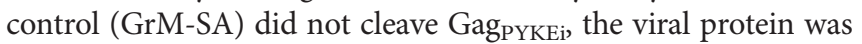
cleaved in a dose- (Figures 1A, B) and time-dependent manner (Figures 1C, D) by GrM. Already at a very low concentration of 5 nM, GrM almost completely degraded full-length Gag PYKEi within one hour. Immunoblotting using an mCherry antibody showed a degradation product of around $29 \mathrm{kDa}$, which could represent the product of mCherry with a small part of the P6 late domain. Immunoblotting using a polyclonal antibody that detects the matrix and capsid domains of Gag revealed degradation products of around $53 \mathrm{kDa}$, which could represent the Gag ${ }_{\text {PYKEi }}$ without the last part of the P6 late domain, and $40 \mathrm{kDa}$. This

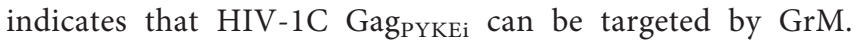
Moreover, the presence of multiple cleavage products suggests that GrM proteolytically processed Gag PYKEi $_{\text {at least at two }}$ different sites.

Next, we examined the ability of GrM to cleave other Gag variants. When we tested GrM-mediated cleavage of Gag variants HIV-1C with a PYQE insertion (HIV-1C Gag PYQEi $_{\text {) }}$ (Figures 2A, B) or without any tetrapeptide insertion (HIV-1C Gag $_{w t}$ ) (Figures 2C, D) and HIV-1B (Figures 2E, F), we observed degradation of Gag with GrM concentrations of 20 $\mathrm{nM}$ or higher. Cleavage is most efficient in the HIV-1C Gag PYKEi variant, which contains the complete consensus GrM substrate

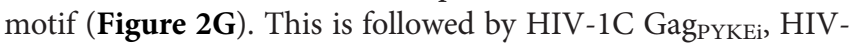
$1 \mathrm{~B}$ and finally HIV-1C wild type in terms of proteolytic efficiency by which GrM can degrade the Gag protein. In conclusion, these data indicate that GrM can cleave Gag regardless of subtype.

\section{Tetrapeptide Insertion PYKE Facilitates Very Efficient GrM-Mediated Cleavage After Leu ${ }^{483}$}

The difference in cleavage efficiency between the tested HIV-1C variants could be caused by the variation in protein sequence at 

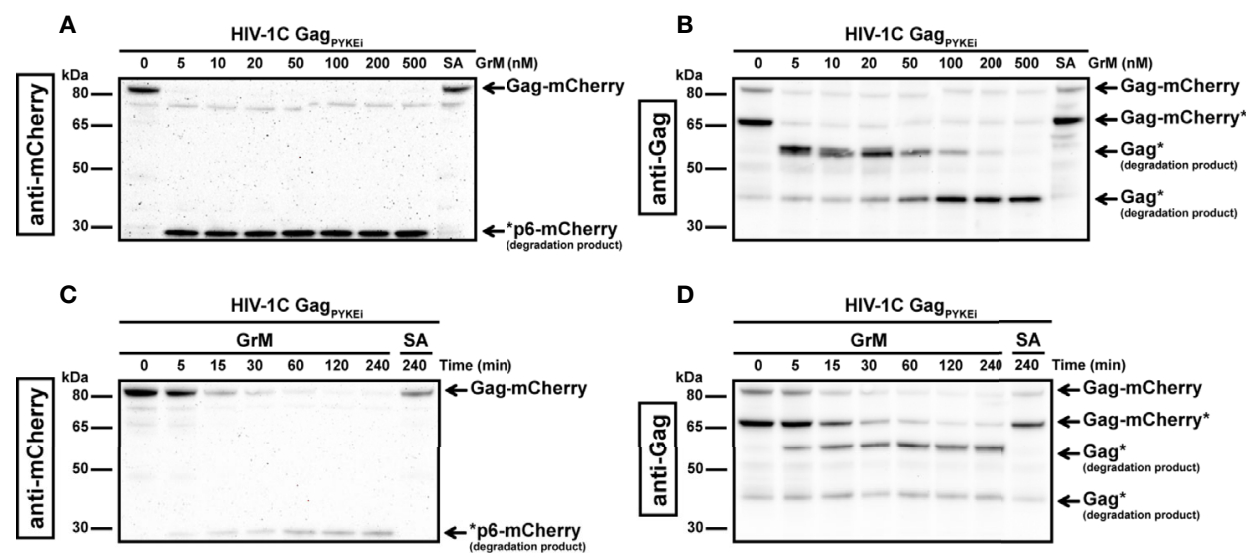

FIGURE 1 | GrM degrades HIV-1 GagpYKEi protein. (A, B) HEK293FT cells were transfected with plasmids encoding for C-terminal mCherry-tagged HIV-1C Gag $_{\mathrm{PYKEi}}$ and lysates $(10 \mu \mathrm{g})$ were incubated with increasing concentrations of GrM or GrM-SA $(500 \mathrm{nM})$ for $4 \mathrm{~h}$ at $37^{\circ} \mathrm{C}$. Samples were subjected to immunoblotting using an anti-mCherry antibody (A) or anti-p55 Gag antibody (B) to detect full length Gag-mCherry and degradation products. (C, D) Lysates (10 $\mu$ g) were incubated with $5 \mathrm{nM}$ of GrM for the indicated time points or GrM-SA for $4 \mathrm{~h}$ at $37^{\circ} \mathrm{C}$ and immunoblotted using an anti-mCherry antibody (C) or anti-p55 Gag antibody (D). Of note, the C-terminal mCherry tag is partially degraded by other cellular proteases as observed by the smaller Gag-mCherry product around $65 \mathrm{kDa}$ (Gag-mCherry*). Data depicted is representable for at least two individual experiments.

the site of the tetrapeptide insertion. The PYKE insertion in HIV-1C Gag creates a complete GrM consensus substrate motif KEPL. Therefore, we used this variant as a tool for our next experiments to identify the main GrM cleavage site and to assess GrM-mediated cleavage of Gag in living cells. First, to test whether GrM can indeed cleave Gag after the predicted leucine residue, we mutated this leucine residue within $\mathrm{Gag}_{\mathrm{PYKEi}}\left(\mathrm{Leu}^{483}\right)$ into an alanine (PYKEi $\left.{ }^{\mathrm{L} 483 \mathrm{~A}}\right)$. HIV-1C-Gag $\mathrm{PYKEi}$ and PYKEi ${ }^{\mathrm{L} 483 \mathrm{~A}}$ were expressed in HEK293FT cells and cell-free protein extracts were incubated with increasing concentrations of GrM (Figures 3A, B). Whereas Gag PYKEi $_{\text {was very efficiently cleaved }}$ by GrM, the cleavage efficiency of the PYKEi ${ }^{\mathrm{L} 483 \mathrm{~A}}$ mutant was significantly lower.

Since we observed that GrM cleaves Gag PYKEi $_{\text {at multiple }}$ sites, albeit at lower efficiencies, we also tested whether GrM cleaves at other potential sites. Based on the size of cleavage products, we predicted there is another cleavage adjacent to the identified $\mathrm{Leu}^{483}$ residue as well as at the end of the matrix region or beginning of the capsid region. We mutated residues Leu ${ }^{101}$, $\mathrm{Leu}^{145}$ or $\mathrm{Leu}^{495}$ within PYKEi ${ }^{\mathrm{L} 483 \mathrm{~A}}$ into an alanine, prepared cell-free protein extracts from cells overexpressing each mutant variant and incubated these extracts with GrM. None of these mutants showed a significant resistance in GrM-mediated cleavage (data not shown). Altogether, Gag PYKEi $_{\text {is most }}$ efficiently cleaved by GrM after Leu ${ }^{483}$ (Figure 3C).

\section{Cytotoxic Lymphocytes Target GagPYKEi Through GrM in Living Cells}

Our previous experiments to analyze GrM-mediated cleavage of Gag were performed in in vitro lysates and purified GrM. Next, we wanted to assess whether GrM could target Gag in a more immunologically relevant setting using co-culture assays with target cells challenged by cytotoxic lymphocytes. To this end, we

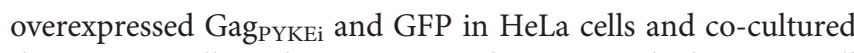
these target cells with IL-2-activated PBMCs, which express all granzymes. After four hours of co-culture, the supernatant was removed, and the remaining adherent cells were directly lysed and subjected to immunoblotting. Since IL-2-activated PBMCs induce non-specific target cell death, but will still be present in our lysates, we used the GFP expression in the HeLa cells as cell viability loading control. Indeed, with increasing effector:target (E:T) ratios, more cell death was induced as observed by the decrease in GFP levels (Figure 4A). No additional decrease in

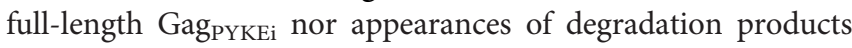
were observed. To limit the bias of cell death in our assay, we performed the experiment in the presence of pan-caspase inhibitor zVAD-FMK. Co-culture in the presence of this pancaspase inhibitor inhibited cell death (Figure 4B). Although there was no clear decrease in full length Gag, we did observe a degradation product of around $\sim 53 \mathrm{kDa}$ similar to the in vitro lysate experiments. Of note, de novo Gag is transported to the membrane where it forms vesicles and is thus constantly released from cells (22), and this could explain why a decrease of fulllength Gag through degradation is not observed.

To have a more GrM-specific co-culture model, we used the natural killer lymphoma KHYG-1 cell line as effector cells instead of IL-2-activated PBMCs in our co-culture assays. Indeed, KHYG-1 cells express high GrM protein levels but very low $\mathrm{GrB}$ protein levels as observed by flow cytometry (data not shown) and immunoblotting (23). In the absence of the pan-caspase inhibitor, the $\sim 53 \mathrm{kDa}$ degradation product was observed in all $\mathrm{E}: \mathrm{T}$ conditions except for the controls (0:1 and 16:0) (Figure 5A). When we performed a time course co-culture at a low E:T ratio $(2: 1)$ in the absence or presence of the pan-caspase inhibitor zVAD-FMK, a clear increase of the degradation product over time was observed in the conditions where $\mathrm{zVAD}-\mathrm{FMK}$ was present (Figure 5B). 

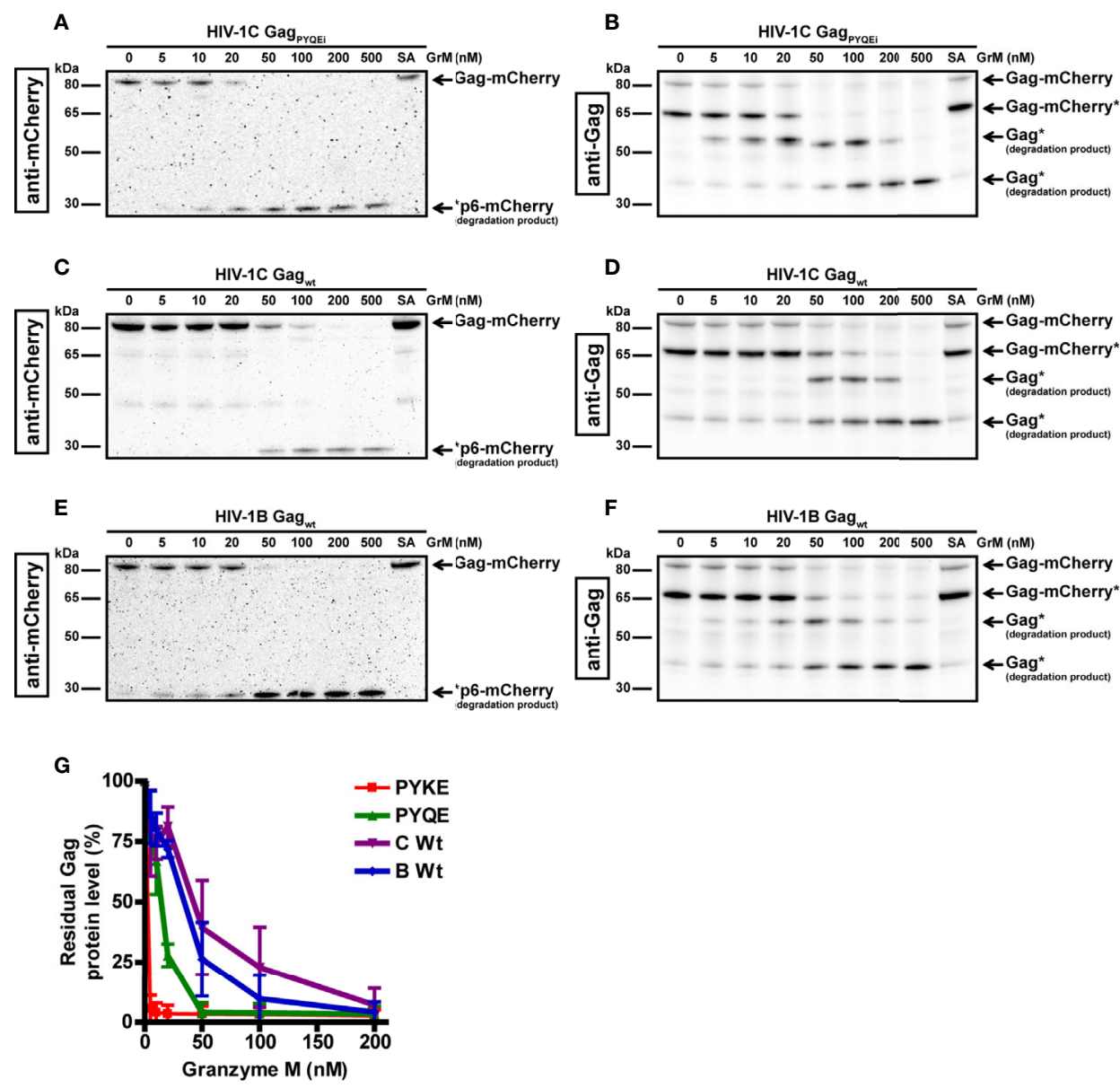

FIGURE 2 | GrM degrades various HIV-1 subtype Gag proteins. HEK293FT cells were transfected with plasmids encoding for C-terminal mCherry-tagged HIV-1C $\operatorname{Gag}_{\mathrm{PYQE}}$ (A, B), HIV-1C GagwT (C, D) or HIV-1B Gagwt (E, F) and lysates $(10 \mu \mathrm{g})$ were incubated with increasing concentrations of GrM or GrM-SA (500 nM) for $4 \mathrm{~h}$ at $37^{\circ} \mathrm{C}$. Samples were subjected to immunoblotting using an anti-mCherry antibody (A, C, E) or anti-p55 Gag antibody (B, D, F) to detect full length GagmCherry and degradation products. (G) Band intensities of full-length Gag-mCherry from all four Gag variants as detected with the anti-mCherry antibody in Figures 1 and 2 and additional experiments were quantified and plotted with Gag incubated with $0 \mathrm{nM}$ GrM set at 100\%. Data points represent the mean \pm SEM from two individual experiments.

Finally, we performed the KHYG-1 co-culture assay in the presence of GrM-inhibitor peptide AcKVPL-CMK

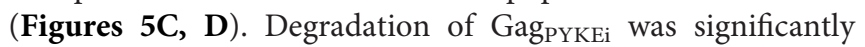
reduced whenever the GrM-inhibitor was present, indicating that this degradation is specifically mediated by GrM. Altogether, these data show that GrM secreted from cytotoxic lymphocytes can cleave Gag in target cells.

\section{GrM Expression Within PBMCs of ECs and VPs}

Since we showed that GrM can target HIV-1 Gag, this could constitute a mechanism for immunological control of HIV-1. ECs are a group of HIV-1 infected individuals who can control their infection in the absence of antiretroviral therapy, and it has been shown that GrM mRNA could be differentially expressed within PBMCs from ECs compared to viremic progressors (VPs) (18). To assess whether differences in GrM levels might be an underlying mechanism by which EC could control HIV-1, we examined both GrM mRNA transcript levels and protein levels within PBMCs from various patient groups. We collected transcriptomics data on an expanded patient cohort and plotted the expression of GrM transcripts in PBMCs for each individual of HIV-1 negative individuals (HCs), ECs, and VPs. GrM transcript expression was highest in HCs, lowered in ECs and lowest in VPs (Figure 6A). However, these differences were not significantly different. Since perforin is required for the intracellular effects of GrM, we also analyzed the transcript levels of perforin (Supplementary Figure 1). However, no differences in perforin transcript levels were observed among the three patient groups. The same analysis was performed for the transcripts of the other four granzymes (Supplementary Figure 1). Like perforin, GrB transcript levels were similar in all three patient groups. The transcript levels of GrA and GrK of ECs were similar to uninfected individuals and increased in VPs. GrH transcripts were increased in ECs and 

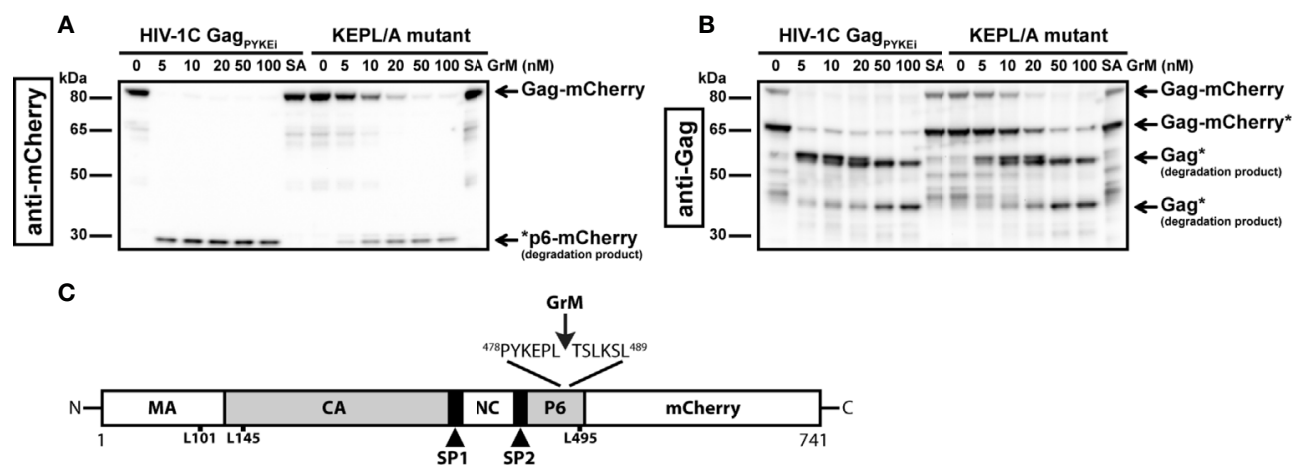

FIGURE 3 | GrM cleaves HIV-1C Gag PYKEi $_{\text {after Leu }}^{483}$. (A, B) Based on the HIV-1C GagPYKEi protein sequence and the GrM acid consensus GrM substrate motif KEPL, we predicted that GrM can cleave GagPYKEi after the leucine residue at position 483 (Leu ${ }^{483}$ ). To test this, we mutated Leu ${ }^{483}$ into an alanine (KEPL/A mutant). Then, HEK293FT cells were transfected with mCherry-tagged HIV-1C GagPYKEi or the KEPL/A mutant. Lysates (10 $\mu \mathrm{g})$ were incubated with increasing concentrations of GrM or GrM-SA (100 nM) for $4 \mathrm{~h}$ at $37^{\circ} \mathrm{C}$ and immunoblotted using an anti-mCherry antibody (A) or anti-p55 Gag antibody (B). (C) Schematic overview of GrM cleavage site within GagPYKEi as well as other tested mutants that were not GrM cleavage sites (MA, p17 matrix protein; CA, p24 capsid protein; SP1, spacer peptide 1; NC, p7 nucleocapsid protein; SP2, spacer peptide 2; P6, p6 late domain).

\section{A} co-culture with PBMCs

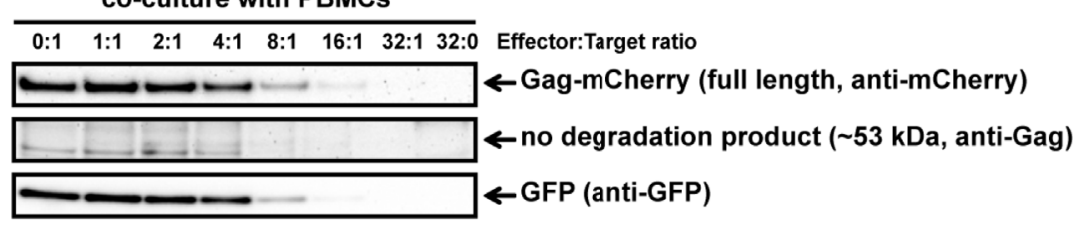

B

co-culture with PBMCs + zVAD-FMK

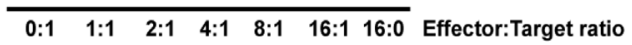

\begin{tabular}{|c|c|}
\hline$-5-5$ & $\leftarrow$ Gag-mCherry (full length, anti-mCherry) \\
\hline 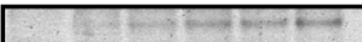 & $\leftarrow$ Gag* $^{*}(\sim 33 \mathrm{kDa}$, anti-Gag) \\
\hline $\bar{E}$ & -GFP (anti-GFP) \\
\hline
\end{tabular}

FIGURE 4 | IL-2-activated PBMCs target Gag PYKE $_{\text {in }}$ ining target cells. (A) HeLa cells were transfected with both mCherry-tagged HIV-1C GagPYKEi and pEGFP-N1 in a 3:1 plasmid ratio and at $24 \mathrm{~h}$ post-transfection these cells were challenged with increasing effector:target (E:T) ratios of IL-2-activated PBMCs for $4 \mathrm{~h}$ at $37^{\circ} \mathrm{C}$. Lysates were subjected to immunoblotting using an anti-mCherry, anti-p55 Gag or anti-GFP antibody. (B) HeLa cells were transfected with both mCherry-tagged HIV-1C GagPYKEi and pEGFP-N1 in a 3:1 plasmid ratio and seeded in the presence of ZVAD-FMK (50 $\mu \mathrm{M})$ or only DMSO (0.5\%). Then, $24 \mathrm{~h}$ post-transfection, these cells were challenged with increasing E:T ratios of IL-2-activated PBMCs in the presence of ZVAD-FMK $(50 \mu \mathrm{M})$ or only DMSO $(0.5 \%)$ for $4 \mathrm{~h}$ at $37^{\circ} \mathrm{C}$. Lysates were subjected to immunoblotting using an anti-mCherry, anti-p55 Gag or anti-GFP antibody.

highest in VPs compared to HCs. Thus, GrM and GrH were the only transcripts differentially expressed in both ECs and VPs compared to the uninfected controls. For all individual granzymes, except for GrK, there was a positive correlation between the granzyme transcript level and perforin transcript levels (Supplementary Figure 2). No correlation was observed between GrM transcript levels with any other individual granzyme transcript levels (Supplementary Figure 3).

Next, we examined the protein expression levels of GrM from HCs, ECs, and VPs (Figure 6B). Here, we observed a reversed expression pattern among the patient groups with the lowest
GrM protein expression in HCs, increased expression in ECs, and highest expression in VPs. The increased protein expression in VPs was significantly different compared to HCs. Similar to the transcriptomics data, perforin protein levels did not differ between the three patient groups (Supplementary Figure 4). In our proteomics data, GrB and GrK were not detected. Protein levels of GrK were following the same trend as for transcript levels; lowest levels in HC, higher levels in EC, and the highest in VP (Supplementary Figure 4). Thus, there is no clear difference in GrM transcript or protein levels between ECs and other patient groups (HCs and VPs). 
A

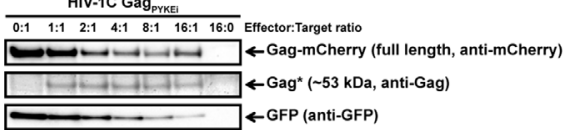

B

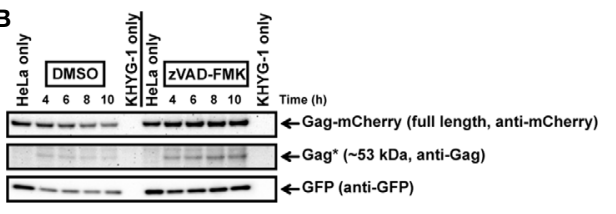

C

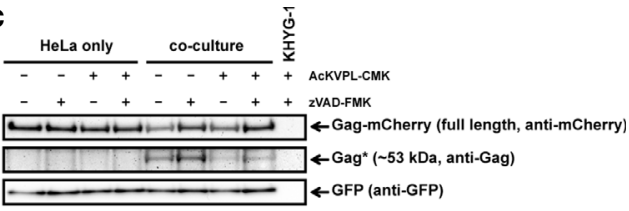

D

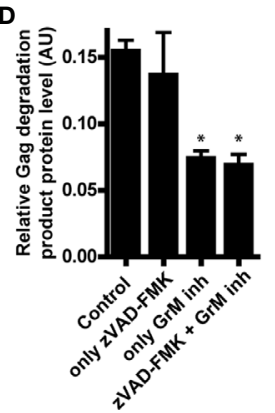

FIGURE 5 | Cytotoxic lymphoma cell line KHYG-1 target GagPYKE in living target cells through GrM. (A) HeLa cells were transfected with both mCherry-tagged HIV-

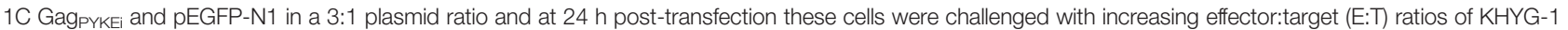
cells for $8 \mathrm{~h}$ at $37^{\circ} \mathrm{C}$. Lysates were subjected to immunoblotting using an anti-mCherry, anti-p55 Gag or anti-GFP antibody. (B) HeLa cells were transfected with both mCherry-tagged HIV-1C GagPYKEi and pEGFP-N1 in a 3:1 plasmid ratio and seeded in the presence of zVAD-FMK (50 $\mu \mathrm{M})$ or only DMSO (0.5\%). Then, $24 \mathrm{~h}$ post-transfection, these cells were challenged with KHYG-1 cells (effector:target ratio of 2:1) in the presence of zVAD-FMK (50 $\mu \mathrm{M})$ or only DMSO (0.5\%) for indicated time points at $37^{\circ} \mathrm{C}$. Lysates were subjected to immunoblotting using an anti-mCherry, anti-p55 Gag or anti-GFP antibody. (C) Co-cultures were performed as in (b) except KHYG-1 cells were pretreated with GrM inhibitor peptide AcKVPL-CMK (100 $\mu \mathrm{M})$ or left untreated for $1 \mathrm{~h}$ at $37^{\circ} \mathrm{C}$ before challenging mCherry-tagged Gag $_{\text {PYKEi }}$ expressing HeLa cell with KHYG-1 cells for $4 \mathrm{~h}$ at $37^{\circ} \mathrm{C}$. (D) Band intensities of the $\sim 53 \mathrm{kDa}$ Gag degradation product as detected with the anti-p55 Gag antibody in (c) and two additional experiments were quantified, and values were normalized for GFP band intensities. Data points are plotted as mean \pm SEM arbitrary units (AU) from triplicate samples. ( ${ }^{*} \mathrm{p}<0.05$ compared to control; ANOVA).

A

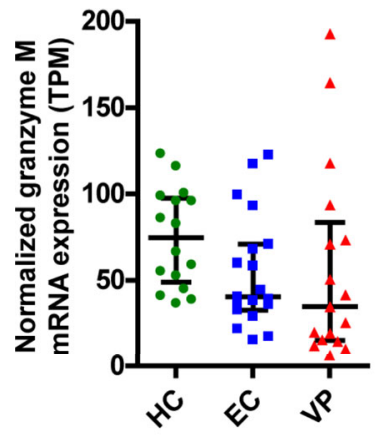

B

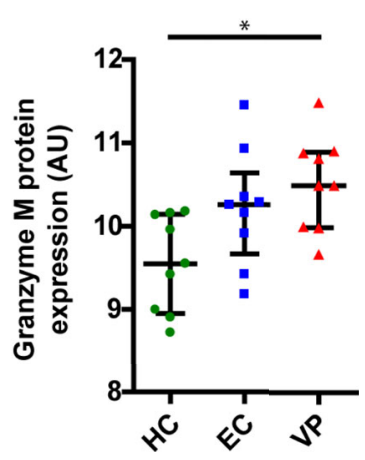

FIGURE 6 | GrM is differentially expressed within PBMCs of individuals from uninfected healthy controls $(\mathrm{HC})$, Elite Controllers $(\mathrm{EC})$ and viral progressors (VP). (A) Normalized expression levels (transcripts per million, TPM) of GrM transcripts within PBMCs from each individual are plotted and median with interquartile range is depicted for each patient group. (B) GrM protein expression (arbitrary units, AU) within PBMCs from each individual are plotted and median with interquartile range is depicted for each patient group. ( ${ }^{*} \mathrm{p}<$ 0.05; Kruskal-Wallis).

\section{DISCUSSION}

Most studies investigating the antiviral response of cytotoxic lymphocytes towards HIV-1-infected cells have focused on GrBpositive $\mathrm{CD}^{+} \mathrm{T}$ lymphocytes. Only a few studies have examined

the role of other granzymes or perforin, or assessed all five human granzymes in HIV-1-specific $\mathrm{CD}^{+} \mathrm{T}$ lymphocytes (11, 24-28). However, these studies only focus on the detection of granzymes within cytotoxic lymphocytes and have not investigated the immunological molecular mechanism by which the granzymes could control HIV-1. Some noncytotoxic antiviral mechanisms employed by granzyme-releasing cytotoxic lymphocytes have been described in the context of other virus infections, such as Herpes simplex virus type 1 (HSV-1), and human cytomegalovirus (HCMV) (8-10). HIV-1 permanently integrates its viral genome into the host cell DNA genome and establish viral latency reservoirs, greatly complicating the possibility to eradicate viruses from the host through cellmediated cytotoxicity by cytotoxic lymphocytes. However, understanding the role of granzymes in counteracting HIV-1 infection, even though noncytotoxic mechanisms, and modulating these activities within cytotoxic lymphocytes could be a strategy to control latent or reactivated HIV-1 infection in the absence of antiretroviral drugs or in individuals failing with low-grade viremia during antiretroviral therapy. In our previous study on HIV-1C Gag variants with or without the tetrapeptide insertion PYxE (16), we noticed a potential GrM cleavage site within Gag, and therefore we studied whether and to what extent GrM can cleave Gag in this study.

Here, we indeed show that GrM targets the HIV-1 Gag protein in its P6 late domain. It has been suggested that Gag can be in a compact conformational structure when in solution or an extended conformational structure when bound to the 
plasma membrane when still at the immature non-processed state, although these observations were demonstrated with Gag molecules that are lacking the P6 domain (29). Gag is predominantly localized at the plasma membrane $(16,30,31)$, and the P6 domain plays an important role prior and during the virion assembly phase (15). Therefore, this domain is exposed to binding to other viral and host cell proteins and thus also exposed for targeting by GrM. Even though only a relatively small $2 \mathrm{kDa}$ C-terminal part of the P6 late domain is being cleaved off, this exposed domain is important for its interaction with host cell protein ALIX and serves as a binding domain for HIV-1 accessory protein Vpr (16, 32-37). The GrM cleavage site is directly after the ALIX binding motif and cleavage could disturb the interaction of ALIX with Gag and thereby affecting the budding of virions from the plasma membrane $(16,33)$. The motif for binding Vpr to Gag, LXSLFG, is located after the GrM cleavage site and deletion of this motif completely abolishes the incorporation of Vpr into virions $(32,34-37)$. Our current model does not take in account the effects of other viral proteins on Gag localization, such as the role that Nef has on Gag (38). Therefore, it would be of great interest to examine to what extent GrMmediated cleavage could inhibit virion release, viral cell-to-cell transfer, or reduce the infectiousness of viral particles.

Although GrM can cleave various variants of Gag, the cleavage efficiency is subtype dependent. Whereas the HIV-1B Gag has a highly conserved amino acid protein sequence near the proline-leucine residues, the HIV-1C Gag shows unique variations with the PYxE tetrapeptide insertions directly before the proline-leucine residues in a subgroup of HIV-1C infected

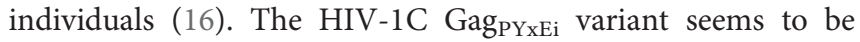
originating from Eastern Africa and is emerging in other countries $(16,39,40)$. The PYKE sequence is most likely the result of a recombination event between HIV-1C that lacks this sequence with HIV-2 that naturally contains the PYKE motif. On the one hand, insertion of the PYKE motif within HIV-1C Gag enhanced its binding capacity to host cell protein ALIX and correlated with increased viral replication and viral fitness (16, 41). On the other hand, PYKE insertion also increases susceptibility towards GrM cleavage. Mutations of the lysine residue into a glutamine (PYQE) or arginine (PYRE) have been observed in different patient cohorts and could be a consequence of immunological pressure $(14,16,42)$. Indeed, Gag ${ }_{\mathrm{PYQEi}}$ shows reduced susceptibility towards GrM-mediated cleavage. These mutations in the tetrapeptide sequence could be a balanced compromise between increased viral replication and degradation by the immune system. Therefore, immunological pressure through a GrM-mediated cytotoxic lymphocyte response could be an important driving factor on the transmission and evolution of HIV-1C Gag PYxEi $_{\text {variants. }}$

Regulation of granzyme expression is most likely different for each individual granzyme. Indeed, GrM is not upregulated in response to cytokines such as IL-2 or IFN $\alpha$ that upregulates other granzymes $(43,44)$. Also, expression of individual granzymes varies among virus-specific $\mathrm{CD}^{+} \mathrm{T}$ lymphocytes. Human cytomegalovirus (HCMV)-specific CD8 ${ }^{+}$T lymphocytes appear to express higher protein levels of GrM compared to
Epstein-Barr Virus- or influenza-specific $\mathrm{CD} 8^{+} \mathrm{T}$ lymphocytes (8). For HIV-1, we observed a decrease in GrM transcript levels in ECs and VPs compared to non-infected individuals, but increased GrM protein levels. The inverse pattern in transcript and protein levels could perhaps indicate a difference in protein turnover and/or release of GrM in cytotoxic lymphocytes. Variations in granzyme levels could also differ in $\mathrm{T}$ lymphocytes of each individual depending on the epitopespecificity and/or HLA-specificity of the T lymphocytes that varies from one individual to another. We do see a wide distribution in GrM transcript levels in VPs, although this could be highly influenced by increased immune activation that occurs in VPs.

We also speculated that differences in GrM levels could be an underlying mechanism in ECs to control their infection. Indeed, in a previous study, GrM transcript levels were significantly different between ECs and VPs, but this study had only 6 VPs included in the analysis (18). Here, we expanded the cohort to include 17 VPs. Although there are minor differences in expression of GrM transcripts and protein levels within ECs compared to HCs and VPs, these differences were not significantly different. Of note, we also did not observe any difference in perforin expression between EC and VP in the total PBMC population, although it has been shown that HIV-1specific $\mathrm{CD}^{+} \mathrm{T}$ cells from ECs does express more perforin upon stimulation with HIV-1 peptide pools (28). Thus, our analysis on whole PBMCs might be not sufficient to detect relevant changes occurring in HIV-1-specific $\mathrm{CD}^{+} \mathrm{T}$ cells. Also, our limited cohort includes individuals infected by one of various subtypes, including A1, B and C (only wild type) (18). It would be worthwhile to examine whether there is a correlation between the subtype you are infected with, including subtype $\mathrm{C}$ with or without the tetrapeptide insertion, and GrM levels within their cytotoxic lymphocytes.

Understanding how the host's immune response could target HIV-1 through GrM exposes weaknesses of HIV-1 infection that could be exploited therapeutically. Although chemical intervention strategies could be designed that mimic the activity of GrM towards the viral Gag protein, immunotherapies exploiting GrM could be a promising alternative approach. HIV-1-specific $\mathrm{CD}^{+} \mathrm{T}$ cells could be genetically modified to increase GrM protein expression and expanded ex vivo. However, ex vivo or expanded HIV-1-specific $\mathrm{CD}^{+} \mathrm{T}$ cells from HIV-1 ECs that are cultured with HIV-1infected $\mathrm{CD}^{+} \mathrm{T}$ cells reduced degranulation activity for all granzyme-positive $\mathrm{CD}^{+} \mathrm{T}$ cells (11). This indicates that merely increasing GrM levels in HIV-1-specific $\mathrm{CD}^{+} \mathrm{T}$ cells would be insufficient to boost anti-HIV-1-responses. Either ex vivo conditions are lacking additional signals that are present at sites of infection to induce $\mathrm{CD}^{+} \mathrm{T}$ cell degranulation or the HIV-1-specific $\mathrm{CD}^{+} \mathrm{T}$ cells are showing signs of exhaustion. Therefore, it will be important to study which additional signals are required for efficient degranulation of genetically modified GrM-positive $\mathrm{CD}^{+} \mathrm{T}$ cells after they have expanded ex vivo. Alternatively, if we could stimulate $\mathrm{HIV}$-1-specific $\mathrm{CD}^{+} \mathrm{T}$ cells to specifically increase GrM protein expression in vivo, we could 
directly utilize the patients' immune response to improve antiHIV-1 activities.

\section{DATA AVAILABILITY STATEMENT}

All RNAseq data are deposited in NCBI under BioProject accession number PRJNA420459. Protein identification results are deposited to the ProteomeXchange Consortium (http:// proteomecentral.proteomexchange.org) via the PRIDE partner repository (PXD023990).

\section{ETHICS STATEMENT}

The study was approved by regional ethics committees of Stockholm (2013/1944-31/4) and amendment (2019-05585). The patients/participants provided their written informed consent to participate in this study.

\section{AUTHOR CONTRIBUTIONS}

ES, FM, PFI, AV, and RvD performed experiments and data analysis. AS, UN, and RvD contributed to conception and design of the study. ES and RvD wrote the manuscript. All authors contributed to the article and approved the submitted version.

\section{REFERENCES}

1. Andrade F. Non-cytotoxic antiviral activities of granzymes in the context of the immune antiviral state. Immunol Rev (2010) 235(1):128-46. doi: 10.1111/ j.0105-2896.2010.00909.x

2. Bovenschen N, Kummer JA. Orphan granzymes find a home. Immunol Rev (2010) 235(1):117-27. doi: 10.1111/j.0105-2896.2010.00889.x

3. Chowdhury D, Lieberman J. Death by a thousand cuts: granzyme pathways of programmed cell death. Annu Rev Immunol (2008) 26:389-420. doi: 10.1146/ annurev.immunol.26.021607.090404

4. van Domselaar R, Bovenschen N. Cell death-independent functions of granzymes: hit viruses where it hurts. Rev Med Virol (2011) 21(5):301-14. doi: 10.1002/rmv.697

5. Mahrus S, Craik CS. Selective chemical functional probes of granzymes A and B reveal granzyme B is a major effector of natural killer cell-mediated lysis of target cells. Chem Biol (2005) 12(5):567-77. doi: 10.1016/j.chembiol.2005.03.006

6. de Poot SA, Lai KW, van der Wal L, Plasman K, Van Damme P, Porter AC, et al. Granzyme M targets topoisomerase II alpha to trigger cell cycle arrest and caspase-dependent apoptosis. Cell Death Differ (2014) 21(3):416-26. doi: 10.1038/cdd.2013.155

7. de Poot SA, Westgeest M, Hostetter DR, Van Damme P, Plasman K, Demeyer K, et al. Human and mouse granzyme $\mathrm{M}$ display divergent and species-specific substrate specificities. Biochem J (2011) 437(3):431-42. doi: 10.1042/BJ20110210

8. van Domselaar R, de Poot SA, Remmerswaal EB, Lai KW, ten Berge IJ, Bovenschen N. Granzyme M targets host cell hnRNP K that is essential for human cytomegalovirus replication. Cell Death Differ (2013) 20(3):419-29. doi: $10.1038 /$ cdd.2012.132

9. van Domselaar R, Philippen LE, Quadir R, Wiertz EJ, Kummer JA, Bovenschen N. Noncytotoxic inhibition of cytomegalovirus replication through NK cell protease granzyme M-mediated cleavage of viral phosphoprotein 71. J Immunol (2010) 185(12):7605-13. doi: 10.4049/ jimmunol.1001503

\section{FUNDING}

The study is funded by grants from the Swedish Research Council (2016-01675 and 2017-05848, AS, https://www.vr.se/), CARE under grant agreement no. 825673 (AS, https://cordis.europa.eu/ project/id/825673), and the Stockholm County Council (ALF 20190451 and CIMED 20200645, AS, https://forskningsstod.vmi. se/), Swedish Research Council Establishment Grant (2017-01330, UN, https://www.vr.se/), The Swedish Physicians Against AIDS Research Foundation (Fob2017-0005, RvD, http://www.aidsfond. se/), Stiftelsen Tornspiran (Sep. 2018, RvD, http:// stiftelsentornspiran.se/), and KI Research Foundation Grant (2018-01640, RvD, https://ki.se/).

\section{ACKNOWLEDGMENTS}

Mass spectrometric analysis and database search for protein identification and quantification were carried out at the Proteomics Biomedicum core facility, Karolinska Institutet, Stockholm.

\section{SUPPLEMENTARY MATERIAL}

The Supplementary Material for this article can be found online at: https://www.frontiersin.org/articles/10.3389/fimmu.2021. 669347/full\#supplementary-material

10. Knickelbein JE, Khanna KM, Yee MB, Baty CJ, Kinchington PR, Hendricks RL. Noncytotoxic lytic granule-mediated CD8+ T cell inhibition of HSV-1 reactivation from neuronal latency. Science (2008) 322(5899):268-71. doi: $10.1126 /$ science.1164164

11. Clayton KL, Collins DR, Lengieza J, Ghebremichael M, Dotiwala F, Lieberman J, et al. Resistance of HIV-infected macrophages to CD8(+) T lymphocytemediated killing drives activation of the immune system. Nat Immunol (2018) 19(5):475-86. doi: 10.1038/s41590-018-0085-3

12. Neogi U, Haggblom A, Santacatterina M, Bratt G, Gisslen M, Albert J, et al. Temporal trends in the Swedish HIV-1 epidemic: increase in non-B subtypes and recombinant forms over three decades. PloS One (2014) 9(6):e99390. doi: 10.1371/journal.pone.0099390

13. Hemelaar J, Elangovan R, Yun J, Dickson-Tetteh L, Fleminger I, Kirtley S, et al. Global and regional molecular epidemiology of HIV-1, 1990-2015: a systematic review, global survey, and trend analysis. Lancet Infect Dis (2019) 19(2):143-55. doi: 10.1016/S1473-3099(18)30647-9

14. Neogi U, Rao SD, Bontell I, Verheyen J, Rao VR, Gore SC, et al. Novel tetrapeptide insertion in Gag-p6 ALIX-binding motif in HIV-1 subtype C associated with protease inhibitor failure in Indian patients. AIDS (2014) 28 (15):2319-22. doi: 10.1097/QAD.0000000000000419

15. Sundquist WI, Krausslich HG. HIV-1 assembly, budding, and maturation. Cold Spring Harb Perspect Med (2012) 2(7):a006924. doi: 10.1101/ cshperspect.a006924

16. van Domselaar R, Njenda DT, Rao R, Sonnerborg A, Singh K, Neogi U. HIV-1 subtype C with PYxE insertion has enhanced binding of Gag-p6 to host cell protein ALIX and increased replication fitness. J Virol (2019) 93(9):e00077-19. doi: $10.1101 / 459156$

17. Chaturbhuj D, Patil A, Gangakhedkar R. PYRE insertion within HIV-1 subtype C p6-Gag functions as an ALIX-dependent late domain. Sci Rep (2018) 8(1):8917. doi: 10.1038/s41598-018-27162-1

18. Zhang W, Ambikan AT, Sperk M, van Domselaar R, Nowak P, Noyan K, et al. Transcriptomics and Targeted Proteomics Analysis to Gain Insights Into the 
Immune-control Mechanisms of HIV-1 Infected Elite Controllers. EBioMedicine (2018) 27:40-50. doi: 10.1016/j.ebiom.2017.11.031

19. Willforss J, Chawade A, Levander F. NormalyzerDE: Online Tool for Improved Normalization of Omics Expression Data and High-Sensitivity Differential Expression Analysis. J Proteome Res (2019) 18(2):732-40. doi: 10.1021/acs.jproteome.8b00523

20. Johnson WE, Li C, Rabinovic A. Adjusting batch effects in microarray expression data using empirical Bayes methods. Biostatistics (2007) 8 (1):118-27. doi: 10.1093/biostatistics/kxj037

21. Ritchie ME, Phipson B, Wu D, Hu Y, Law CW, Shi W, et al. limma powers differential expression analyses for RNA-sequencing and microarray studies. Nucleic Acids Res (2015) 43(7):e47-e. doi: 10.1093/nar/gkv007

22. Jouvenet N, Bieniasz PD, Simon SM. Imaging the biogenesis of individual HIV-1 virions in live cells. Nature (2008) 454(7201):236-40. doi: 10.1038/nature06998

23. Suck G, Branch DR, Smyth MJ, Miller RG, Vergidis J, Fahim S, et al. KHYG-1, a model for the study of enhanced natural killer cell cytotoxicity. Exp Hematol (2005) 33(10):1160-71. doi: 10.1016/j.exphem.2005.06.024

24. Bengsch B, Ohtani T, Khan O, Setty M, Manne S, O’Brien S, et al. EpigenomicGuided Mass Cytometry Profiling Reveals Disease-Specific Features of Exhausted CD8 T Cells. Immunity (2018) 48(5):1029-45 e5. doi: 10.1016/ j.immuni.2018.04.026

25. Harari A, Bellutti Enders F, Cellerai C, Bart PA, Pantaleo G. Distinct profiles of cytotoxic granules in memory CD8 T cells correlate with function, differentiation stage, and antigen exposure. J Virol (2009) 83(7):2862-71. doi: 10.1128/JVI.02528-08

26. Migueles SA, Osborne CM, Royce C, Compton AA, Joshi RP, Weeks KA, et al. Lytic granule loading of CD8+ $\mathrm{T}$ cells is required for HIV-infected cell elimination associated with immune control. Immunity (2008) 29(6):100921. doi: 10.1016/j.immuni.2008.10.010

27. Chen G, Shankar P, Lange C, Valdez H, Skolnik PR, Wu L, et al. CD8 T cells specific for human immunodeficiency virus, Epstein-Barr virus, and cytomegalovirus lack molecules for homing to lymphoid sites of infection. Blood (2001) 98(1):156-64. doi: 10.1182/blood.V98.1.156

28. Hersperger AR, Pereyra F, Nason M, Demers K, Sheth P, Shin LY, et al. Perforin expression directly ex vivo by HIV-specific CD8 T-cells is a correlate of HIV elite control. PloS Pathog (2010) 6(5):e1000917. doi: 10.1371/ journal.ppat.1000917

29. Munro JB, Nath A, Farber M, Datta SA, Rein A, Rhoades E, et al. A conformational transition observed in single HIV-1 Gag molecules during in vitro assembly of virus-like particles. J Virol (2014) 88(6):3577-85. doi: 10.1128/JVI.03353-13

30. Jouvenet N, Neil SJ, Bess C, Johnson MC, Virgen CA, Simon SM, et al. Plasma membrane is the site of productive HIV-1 particle assembly. PloS Biol (2006) 4 (12):e435. doi: 10.1371/journal.pbio.0040435

31. Ono A, Freed EO. Cell-type-dependent targeting of human immunodeficiency virus type 1 assembly to the plasma membrane and the multivesicular body. J Virol (2004) 78(3):1552-63. doi: 10.1128/JVI.78.3.1552-1563.2004

32. Bachand F, Yao X-J, Hrimech M, Rougeau N, Cohen ÉA. Incorporation of Vpr into Human Immunodeficiency Virus Type 1 Requires a Direct Interaction with the p6 Domain of the p55 Gag Precursor. J Biol Chem (1999) 274(13):9083-91. doi: 10.1074/jbc.274.13.9083

33. Fisher RD, Chung H-Y, Zhai Q, Robinson H, Sundquist WI, Hill CP. Structural and Biochemical Studies of ALIX/AIP1 and Its Role in Retrovirus Budding. Cell (2007) 128(5):841-52. doi: 10.1016/j.cell.2007.01.035
34. Kondo E, Göttlinger HG. A conserved LXXLF sequence is the major determinant in p6gag required for the incorporation of human immunodeficiency virus type 1 Vpr. J Virol (1996) 70(1):159. doi: 10.1128/ JVI.70.1.159-164.1996

35. Kondo E, Mammano F, Cohen EA, Göttlinger HG. The p6gag domain of human immunodeficiency virus type 1 is sufficient for the incorporation of Vpr into heterologous viral particles. J Virol (1995) 69(5):2759. doi: 10.1128/ JVI.69.5.2759-2764.1995

36. Lu YL, Bennett RP, Wills JW, Gorelick R, Ratner L. A leucine triplet repeat sequence (LXX)4 in p6gag is important for Vpr incorporation into human immunodeficiency virus type 1 particles. J Virol (1995) 69(11):6873. doi: 10.1128/JVI.69.11.6873-6879.1995

37. Paxton W, Connor RI, Landau NR. Incorporation of Vpr into human immunodeficiency virus type 1 virions: requirement for the p6 region of gag and mutational analysis. J Virol (1993) 67(12):7229. doi: 10.1128/ JVI.67.12.7229-7237.1993

38. Malbec M, Sourisseau M, Guivel-Benhassine F, Porrot F, Blanchet F, Schwartz O, et al. HIV-1 Nef promotes the localization of Gag to the cell membrane and facilitates viral cell-to-cell transfer. Retrovirology (2013) 10:80. doi: 10.1186/ 1742-4690-10-80

39. Amogne W, Bontell I, Grossmann S, Aderaye G, Lindquist L, Sönnerborg A, et al. Phylogenetic Analysis of Ethiopian HIV-1 Subtype C Near Full-Length Genomes Reveals High Intrasubtype Diversity and a Strong Geographical Cluster. AIDS Res Hum Retroviruses (2016) 32(5):471-4. doi: 10.1089/ aid.2015.0380

40. Tully DC, Wood C. Chronology and evolution of the HIV-1 subtype C epidemic in Ethiopia. AIDS (2010) 24(10):1577-82. doi: 10.1097/ QAD.0b013e32833999e1

41. Ajasin DO, Rao VR, Wu X, Ramasamy S, Pujato M, Ruiz AP, et al. CCL2 mobilizes ALIX to facilitate Gag-p6 mediated HIV-1 virion release. eLife (2019) 8:e35546. doi: 10.7554/eLife.35546

42. Neogi U, Engelbrecht S, Claassen M, Jacobs GB, van Zyl G, Preiser W, et al. Mutational Heterogeneity in p6 Gag Late Assembly (L) Domains in HIV-1 Subtype C Viruses from South Africa. AIDS Res Hum Retroviruses (2015) 32 (1):80-4. doi: 10.1089/aid.2015.0266

43. de Koning PJA, Tesselaar K, Bovenschen N, Çolak S, Quadir R, Volman TJH, et al. The cytotoxic protease granzyme $M$ is expressed by lymphocytes of both the innate and adaptive immune system. Mol Immunol (2010) 47(4):903-11. doi: 10.1016/j.molimm.2009.10.001

44. Zhang B, Zhang J, Tian Z. Comparison in the effects of IL-2, IL-12, IL-15 and IFNQ on gene regulation of granzymes of human NK cell line NK-92. Int Immunopharmacol (2008) 8(7):989-96. doi: 10.1016/j.intimp.2008.03.001

Conflict of Interest: The authors declare that the research was conducted in the absence of any commercial or financial relationships that could be construed as a potential conflict of interest.

Copyright @ 2021 Saccon, Mikaeloff, Figueras Ivern, Végvári, Sönnerborg, Neogi and van Domselaar. This is an open-access article distributed under the terms of the Creative Commons Attribution License (CC BY). The use, distribution or reproduction in other forums is permitted, provided the original author(s) and the copyright owner(s) are credited and that the original publication in this journal is cited, in accordance with accepted academic practice. No use, distribution or reproduction is permitted which does not comply with these terms. 